This item was submitted to Loughborough's Research Repository by the author.

Items in Figshare are protected by copyright, with all rights reserved, unless otherwise indicated.

\title{
A simple and effective element distortion factor
}

PLEASE CITE THE PUBLISHED VERSION

PUBLISHER

(C) Elsevier

VERSION

AM (Accepted Manuscript)

LICENCE

CC BY-NC-ND 4.0

REPOSITORY RECORD

El-Hamalawi, Ashraf. 2019. "A Simple and Effective Element Distortion Factor". figshare. https://hdl.handle.net/2134/5161. 
This item was submitted to Loughborough's Institutional Repository (https://dspace.lboro.ac.uk/) by the author and is made available under the following Creative Commons Licence conditions.

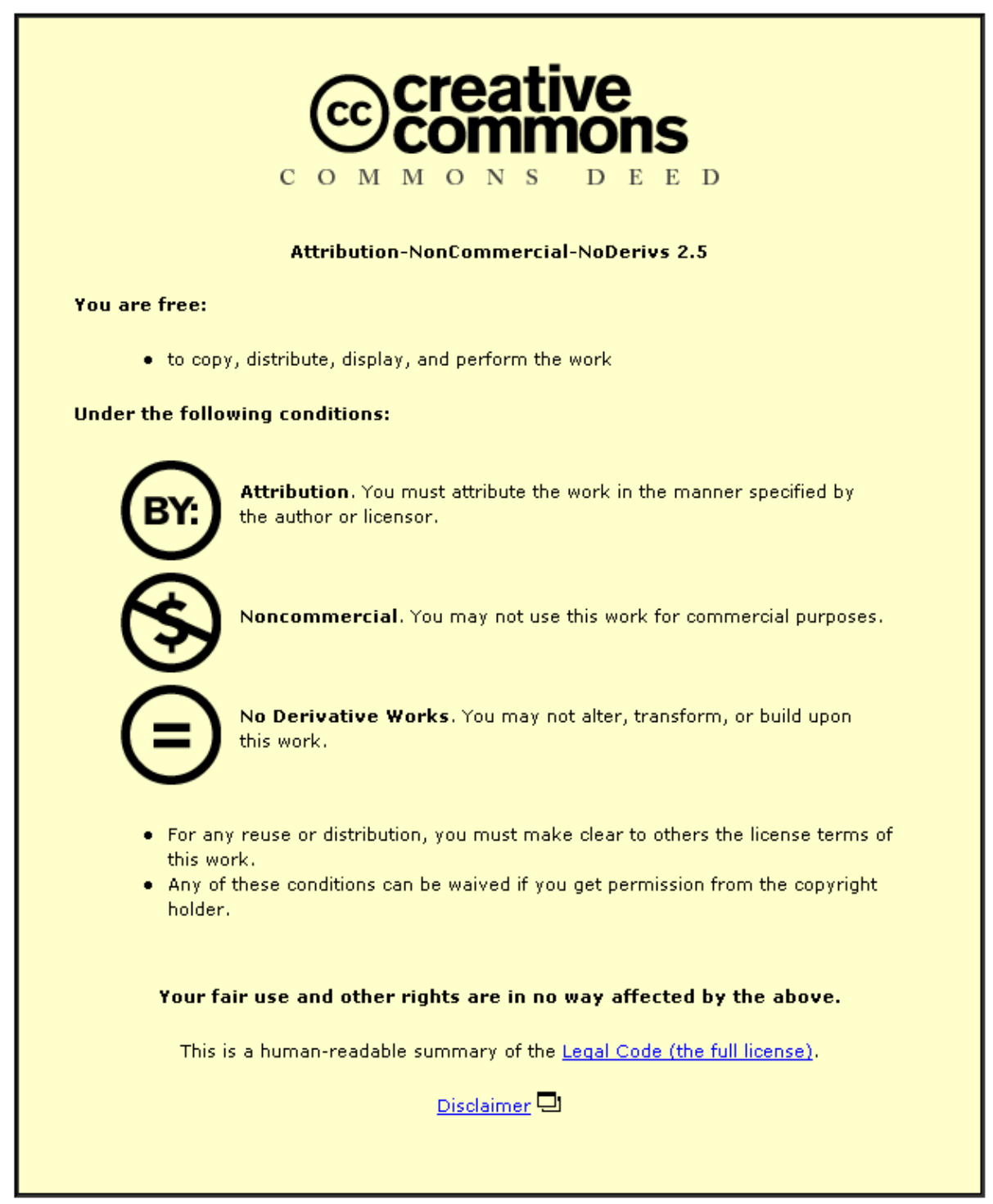

For the full text of this licence, please go to: http://creativecommons.org/licenses/by-nc-nd/2.5/ 


\title{
A SIMPLE AND EFFECTIVE ELEMENT DISTORTION
}

\section{FACTOR}

\author{
A. El-Hamalawi* \\ IGT, Swiss Federal Institute of Technology (ETH Hönggerberg), CH-8093 \\ Zürich, Switzerland
}

\begin{abstract}
In this paper, the main methods employed during mesh generation, in order to control element distortion, are reviewed, and some shortcomings are outlined. A new distortion factor is then introduced, which is both simple to implement, and provides a rapid measure of an element's quality. Some meshes demonstrating their use are finally presented.
\end{abstract}

Keywords : element quality, element distortion factor, mesh generation

\section{INTRODUCTION}

One of the main concerns when generating a new mesh is the quality of elements produced. This becomes especially important for triangle-based quadrilateral mesh generators, such as in References [1, 2]. The use of element distortion factors is therefore a necessary step in order to ensure that the elements' shapes do not act as an extra cause of deterioration in finite element analysis accuracy.

\footnotetext{
* Formerly Engineering Department, Cambridge University, Trumpington Street, Cambridge CB2 1PZ, U.K.
} 
Element shape distortion can be defined in many forms, most notable of which are aspect ratios, skew, taper and warp. Robinson [3] used three of the latter properties to classify the distortion of 4-noded quadrilaterals. A sensible choice of distortion measure would be the determinant of the element's Jacobian matrix. Robinson expressed the shape parameters in terms of the Jacobian determinant for 4-noded quadrilaterals. However, the difficulty arising in linking each element's Jacobian determinant to such parameters (e.g. the aspect ratio or internal angles) for elements of displacement order higher than one with curved sides was pointed out. Eight further parameters in the form of the tangential and normal deviations for each of the four edges were later added [4]. Although these twelve parameters for 8-noded curved quadrilaterals provide a high degree of accuracy, a higher computational workload is needed when calculating all twelve factors for each element. Comparison between various elements would also be difficult unless a factor incorporating all twelve parameters is developed. Most finite element users avoid curved quadrilaterals in mesh generation except at domain boundaries, and prefer to rely on straight-edged elements irrespective of the element order, due to the rapid deterioration in element performance.

Lo [1,5] proposed using a triangular quality factor $\alpha$, defined by equation (1) for a triangle having vertices $\mathrm{A}, \mathrm{B}$ and $\mathrm{C}$, whereby an increase in $\alpha$ produces a better quality triangle. The $2 \sqrt{3}$ is a normalising factor allowing an equilateral triangle, which is the perfect shape for a triangle, to have an $\alpha$ value of unity. A value of $\alpha$ equal to zero corresponds to A, B and C being co-linear.

$$
\alpha=2 \sqrt{3} \frac{\|C A \times C B\|}{\|A B\|^{2}+\|B C\|^{2}+\|C A\|^{2}}
$$

Lo [1] also defined a distortion coefficient $\beta$ in the form of equation (2), for quadrilaterals based on the triangular coefficient $\alpha$. A given quadrilateral ABCD is cut along the two diagonals AC and BD into four triangles, and their corresponding $\alpha_{\mathrm{i}}$ are calculated. 
The higher the value of $\beta$, the less the distortion of the quadrilateral, ranging from unity for squares, where the latter is the perfect quadrilateral shape, to zero if the quadrilateral degenerates to a triangle.

$$
\beta=\frac{\alpha_{3} \alpha_{4}}{\alpha_{1} \alpha_{2}}, \alpha_{1} \geq \alpha_{2} \geq \alpha_{3} \geq \alpha_{4}, \alpha_{i}=\left\{\alpha_{A B C}, \alpha_{A C D}, \alpha_{A B D}, \alpha_{B C D}\right\}
$$

Lau and Lo [6] recently defined a factor $\gamma$ as the ratio of the actual element size to the required element size. The latter authors suggested that any $\gamma$ value between 0.95 and 1.05, i.e. $5 \%$ deviation from the actual element size, should be considered as almost perfect. This becomes important when using density-based mesh generators in order to check that the elements generated are within the densities specified. However, this factor does not provide a measure of how distorted an element is, and another factor would have to be used in conjunction with $\gamma$ to ensure a satisfactory element shape.

Zhu et al. [7] deemed a quadrilateral element satisfactory if all its internal angles $\theta$ fell within $90^{\circ} \pm 45^{\circ}$ and was considered as unsatisfactory if $\theta$ exceeded the limits $90^{\circ} \pm 60^{\circ}$. Lo and Lee [2] found that the first condition appeared to be too strict a condition, so a more flexible range of $90^{\circ} \pm 52.5^{\circ}$ was used for quadrilateral interior angles.

From surveying the literature, it seems that Lo’s triangular and quadrilateral factors are the element shape factors most commonly used. However, even though these factors have been very useful in ensuring high quality elements are generated $[8,9]$, a shortcoming of the quadrilateral factor will be outlined in the next section. New element shape factors for triangles and quadrilaterals are therefore devised, which are easy to visualise and which relate directly to the element interior angles, simultaneously facilitating a comparison with the angle ranges defined by Zhu et al. [7].

\section{DISTORTION FACTORS}


As mentioned previously, the optimum shapes for quadrilaterals and triangles are squares and equilaterals, with interior angles of $90^{\circ}$ and $60^{\circ}$ respectively. The main objective would therefore be to minimise the deviation $\delta \theta_{\mathrm{i}}$ for each interior angle $\theta_{\mathrm{i}}$, defined by equation (3). If the deviations $\delta \theta_{i}$ were thought of as components of a vector $\vec{f}$, the length of $\vec{f}$ being defined by its norm, then the shorter the length of $\vec{f}$, the better the element's shape. Shape factors $\left\|\overrightarrow{\mathrm{f}}_{\mathrm{Q}}\right\|$ and $\left\|\overrightarrow{\mathrm{f}}_{\mathrm{T}}\right\|$, defined by equations (4) and (5), are therefore proposed as quality measures for quadrilaterals and triangles respectively, where $\hat{\mathrm{e}}_{\mathrm{i}}$ are unit vectors.

$$
\begin{gathered}
\delta \theta_{\mathrm{Q}}=\left|\frac{\pi}{2}-\theta_{i}\right| \text { for quadrilaterals, } \delta \theta_{\mathrm{T}}=\left|\frac{\pi}{3}-\theta_{i}\right| \text { for triangles } \\
\overrightarrow{\mathrm{f}}_{\mathrm{Q}}=\delta \theta_{1} \hat{\mathrm{e}}_{1}+\delta \theta_{2} \hat{\mathrm{e}}_{2}+\delta \theta_{3} \hat{\mathrm{e}}_{3}+\delta \theta_{4} \hat{\mathrm{e}}_{4},\left\|\overrightarrow{\mathrm{f}}_{\mathrm{Q}}\right\|=\sqrt{\sum_{\mathrm{i}=1}^{4}\left(\delta \theta_{\mathrm{i}}\right)^{2}} \\
\overrightarrow{\mathrm{f}}_{\mathrm{T}}=\delta \theta_{1} \hat{\mathrm{e}}_{1}+\delta \theta_{2} \hat{\mathrm{e}}_{2}+\delta \theta_{3} \hat{\mathrm{e}}_{3},\left\|\overrightarrow{\mathrm{f}}_{\mathrm{T}}\right\|=\sqrt{\sum_{\mathrm{i}=1}^{3}\left(\delta \theta_{\mathrm{i}}\right)^{2}}
\end{gathered}
$$

It can be seen that $\left\|\overrightarrow{\mathrm{f}}_{\mathrm{Q}}\right\|$ would attain a minimum value of zero for a perfect square and the acceptable range of $90^{\circ} \pm 45^{\circ}$ defined by Zhu et al. [7] would correspond to $\left(\delta \theta_{\mathrm{i}}\right)_{\max }$ equal to $\pi / 4$, or $\left\|\overrightarrow{\mathrm{f}}_{\mathrm{Q}}\right\| \leq \pi / 2$. Similarly, $\left\|\overrightarrow{\mathrm{f}}_{\mathrm{T}}\right\|$ 's minimum value is zero for a perfect equilateral, and an arbitrary value of $60^{\circ} \pm 30^{\circ}$ for triangles would lead to $\left(\delta \theta_{\mathrm{i}}\right)_{\max }$ equal to $\pi / 6$, or $\left\|\overrightarrow{\mathrm{f}}_{\mathrm{T}}\right\| \leq \pi / \sqrt{12}$.

For a hybrid mesh comprising triangles and quadrilaterals, Lo and Lee $[2,10]$ devised a combined quality factor $\mu$, defined in equations (6) and (7), which in mathematical terms is the geometric mean of the $\alpha$ and $\beta$ values of the elements in the mesh.

$$
\mu=\left(\beta^{\mathrm{NQ}} \alpha^{\mathrm{NT}}\right)^{\frac{1}{\mathrm{NQ}+\mathrm{NT}}}
$$




$$
\begin{aligned}
& \alpha=\left\{\begin{array}{cc}
\left(\prod_{\mathrm{i}=1}^{\mathrm{NT}} \alpha_{i}\right)^{\frac{1}{\mathrm{NT}}} & \text { if } \mathrm{NT}>0 \\
1 & \text { if } \mathrm{NT}=0
\end{array}\right. \\
& \beta=\left\{\begin{array}{cc}
\left(\prod_{\mathrm{j}=1}^{\mathrm{NQ}} \beta_{j}\right)^{\frac{1}{\mathrm{NQ}}} & \text { if NQ }>0 \\
1 & \text { if } \mathrm{NQ}=0
\end{array}\right.
\end{aligned}
$$

NQ and NT are the number of quadrilaterals and triangles in the mesh, and $\alpha_{i}$ and $\beta_{\mathrm{j}}$ are the quality factors of the $i$ th triangle and $j$ th quadrilateral respectively. If $\alpha$ and $\beta$ are greater than 0.87 and 0.54 respectively, the triangular and quadrilateral meshes are considered to be of good quality, and greater than 0.95 and 0.72 respectively, then the mesh quality is excellent. An arbitrary value of $\mu$ equal to 0.69 was chosen by Lo and Lee as an indication of a good hybrid mesh. The geometric means $\left\|\overrightarrow{\mathrm{f}}_{\mathrm{Q}}\right\|$ and $\left\|\overrightarrow{\mathrm{f}}_{\mathrm{T}}\right\|$ for the mesh cannot be calculated in a similar way to equation (7), since in contrast to Lo's parameters, $\left\|\overrightarrow{\mathrm{f}}_{\mathrm{Q}}\right\|$ and $\left\|\overrightarrow{\mathrm{f}}_{\mathrm{T}}\right\|$ are close to zero for good quality elements. The latter parameters for the mesh are thus calculated and reported as arithmetic means. The minimum and maximum values of $\left\|\overrightarrow{\mathrm{f}}_{\mathrm{Q}}\right\|$ and $\left\|\overrightarrow{\mathrm{f}}_{\mathrm{T}}\right\|$ for the various meshes have also been included in the examples' section. However, there is no direct way of comparing the values with Lo and Lee's values, so the $\left\|\overrightarrow{\mathrm{f}}_{\mathrm{Q}}\right\|$ and $\left\|\overrightarrow{\mathrm{f}}_{\mathrm{T}}\right\|$ factors are converted into angle ranges using equations (8) and (9) for comparison purposes in the examples' section.

$$
\begin{aligned}
& \left\|\overrightarrow{\mathrm{f}}_{\mathrm{Q}}\right\|=\sqrt{\sum_{\mathrm{i}=1}^{4}\left(\delta \theta_{\mathrm{i}}\right)^{2}}=2 \delta \theta_{\mathrm{Q}} \Rightarrow \delta \theta_{\mathrm{Q}}=\frac{\left\|\overrightarrow{\mathrm{f}}_{\mathrm{Q}}\right\|}{2} \\
& \left\|\overrightarrow{\mathrm{f}}_{\mathrm{T}}\right\|=\sqrt{\sum_{\mathrm{i}=1}^{3}\left(\delta \theta_{\mathrm{i}}\right)^{2}}=\sqrt{3} \delta \theta_{\mathrm{T}} \Rightarrow \delta \theta_{\mathrm{T}}=\frac{\left\|\overrightarrow{\mathrm{f}}_{\mathrm{T}}\right\|}{\sqrt{3}} \\
& \text { quadrilateral angle range }=\frac{\pi}{2} \pm \delta \theta_{\mathrm{Q}} \text { (rad) }
\end{aligned}
$$




$$
\text { triangle angle range }=\frac{\pi}{3} \pm \delta \theta_{\mathrm{T}}(\mathrm{rad})
$$

This author has subjected Lo's quadrilateral and triangle factors to a test, where factors for a large range of angles were calculated and compared. It was found that for certain angle ranges, the quadrilateral factors were inconsistent. As an example, a trapezium with internal angles of $10^{\circ}, 10^{\circ}, 170^{\circ}$ and $170^{\circ}$ and dimensions shown in Figure 1(a), have Lo’s factor $\beta$ equal to 0.969 , while $\left\|\vec{f}_{\mathrm{Q}}\right\|$ equals 2.793 . It can be seen that Lo's quality factor is obviously in error even though Lo classified a quadrilateral with $\beta$ equal to 0.72 as being excellent. The new factor $\left\|\vec{f}_{\mathrm{Q}}\right\|$ however corresponds to $\left(\delta \theta_{\mathrm{i}}\right)_{\max }$ equal to $4 \pi / 9$, i.e. a deviation of $80^{\circ}$. Similarly, a quadrilateral having angles $88^{\circ}, 92^{\circ}, 88^{\circ}$ and $92^{\circ}$ as shown in Figure 1(b) also had the same value of $\beta .\left\|\vec{f}_{\mathrm{Q}}\right\|$ in this case is equal to 0.035 , corresponding to $\left(\delta \theta_{\mathrm{i}}\right)_{\max }$ equal to $\pi / 90$, i.e. a deviation of $2^{\circ}$. It can thus be seen that $\left\|\vec{f}_{\mathrm{Q}}\right\|$ provides more consistent results than Lo's quadrilateral quality factor throughout all quadrilateral angles. One important point is the dependency of Lo's factor on the element's length, and in turn the aspect ratio. For the above example, this was equal to six, which is regarded as an acceptable aspect ratio [11, 12]. Various aspect ratios were included in comparing the factors, and a similar inconsistency was observed for several other angles outside the acceptable interior angle range. The aspect ratios should not pose any problems when using a density-based mesh generator, where element sizes are controlled and aspect ratios are within acceptable ranges. This inconsistency was not found with Lo's triangular factor, which was of comparable accuracy to $\left\|\overrightarrow{\mathrm{f}}_{\mathrm{T}}\right\|$. 


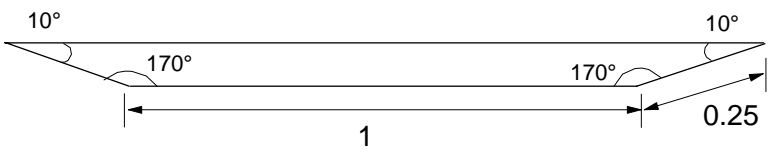

(a) Distorted quadrilateral with interior angles of $90^{\circ} \pm 80^{\circ}$

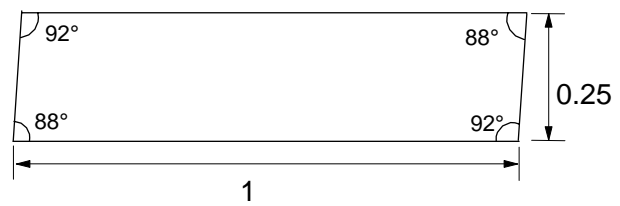

(b) Acceptable quadrilateral with interior angles of $90^{\circ} \pm 10^{\circ}$

\section{Figure 1. Two cases demonstrating an inconsistency using $\beta$}

\section{EXAMPLES}

In this section, the new distortion factors are calculated for typical triangles and quadrilaterals. The quality of the elements generated for two meshes are then assessed using the quality factors, and converted to interior element angle ranges to demonstrate their use. The extra time required to compute the distortion factors is also measured, and compared with the total mesh generation time. Sudden changes in the element sizes have been included in the examples by using adaptive mesh refinement, in order to generate elements with a variety of interior angles and distortion factors.

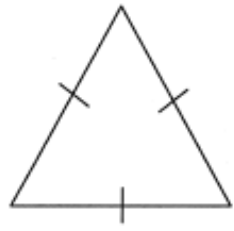

$\alpha=1$ $\left\|\vec{f}_{\mathrm{T}}\right\|=0$

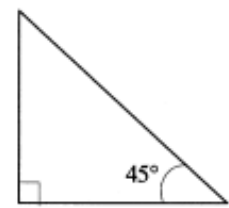

$\alpha=0.866$

$\left|\vec{f}_{\mathrm{T}}\right|=0.641$

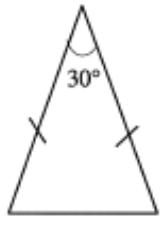

$\alpha=0.764$

$\left\|\overrightarrow{\mathrm{f}}_{\mathrm{T}}\right\|=0.641$

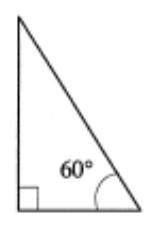

$\alpha=0.75$

$\left|\vec{f}_{\mathrm{T}}\right|=0.740$

(a) Typical triangles

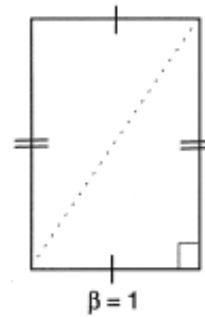

$\left|\hat{f}_{e}\right|=0$

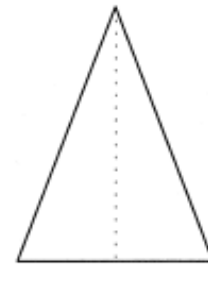

$\beta=0$

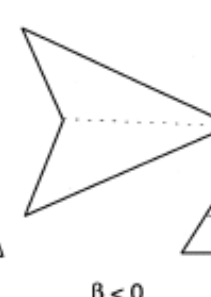

$\beta<0$

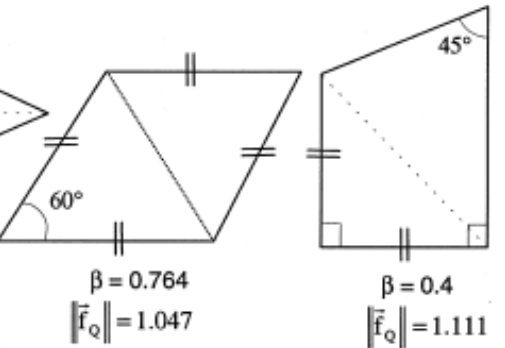

(b) Typical quadrilaterals

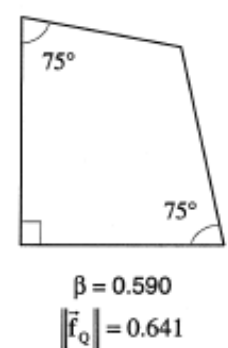

$\left|\overrightarrow{\mathrm{f}}_{\mathrm{Q}}\right|=0.641$

Figure 2. Distortion factors for typical (a) triangles and (b) quadrilaterals 
Figure 2(a) shows some typical triangles with the new distortion factors computed and compared with Lo's $\alpha$ factors. The quadrilaterals presented in Figure 2(b) are elements that could result from typical triangle-based quadrilateral mesh generators. Although Lo's $\beta$ values have been computed for the second and third quadrilaterals, the calculation of $\left\|\vec{f}_{\mathrm{Q}}\right\|$ values would be erroneous. However, a simple safeguard to detect such elements would be to ensure that all four quadrilateral internal angles exist, and are all less than $180^{\circ}$.

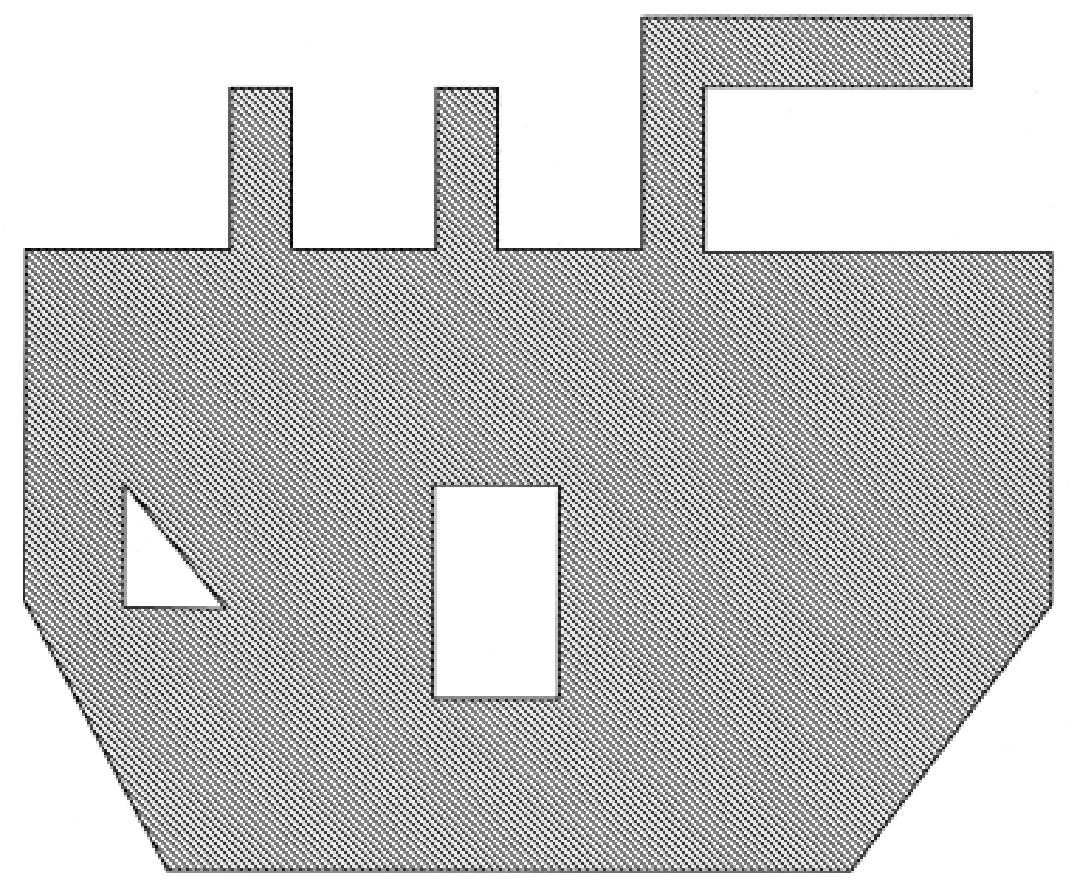

Figure 3. Domain with internal openings and narrow edges

Figure 3 depicts a machine part with two holes of different shapes undergoing various loads. Initially, a purely triangular mesh was produced, shown in Figure 4, which consisted of 1221 elements. The distortion factors and consumed times are also shown in Table 1. As can be seen, the construction of the mesh required a total CPU time of 13.4 seconds. The calculation of the new distortion factors for each element, and comparing it with a prescribed limit, required 0.21 seconds. This is extremely small compared to the time required for the mesh generation and finite element analysis itself. This was also confirmed for several other 
meshes tested. The maximum and minimum triangular quality factors $\left\|\overrightarrow{\mathrm{f}}_{\mathrm{T}}\right\|$ in the mesh were found to be equal to 1.013 and 0.004 , corresponding to angle ranges of $60^{\circ} \pm 23.5^{\circ}$ and $60^{\circ} \pm 0.1^{\circ}$ respectively. These are excellent angle ranges, with the overall mean mesh quality factor 0.376 corresponding to an angle range of $60^{\circ} \pm 12.4^{\circ}$.

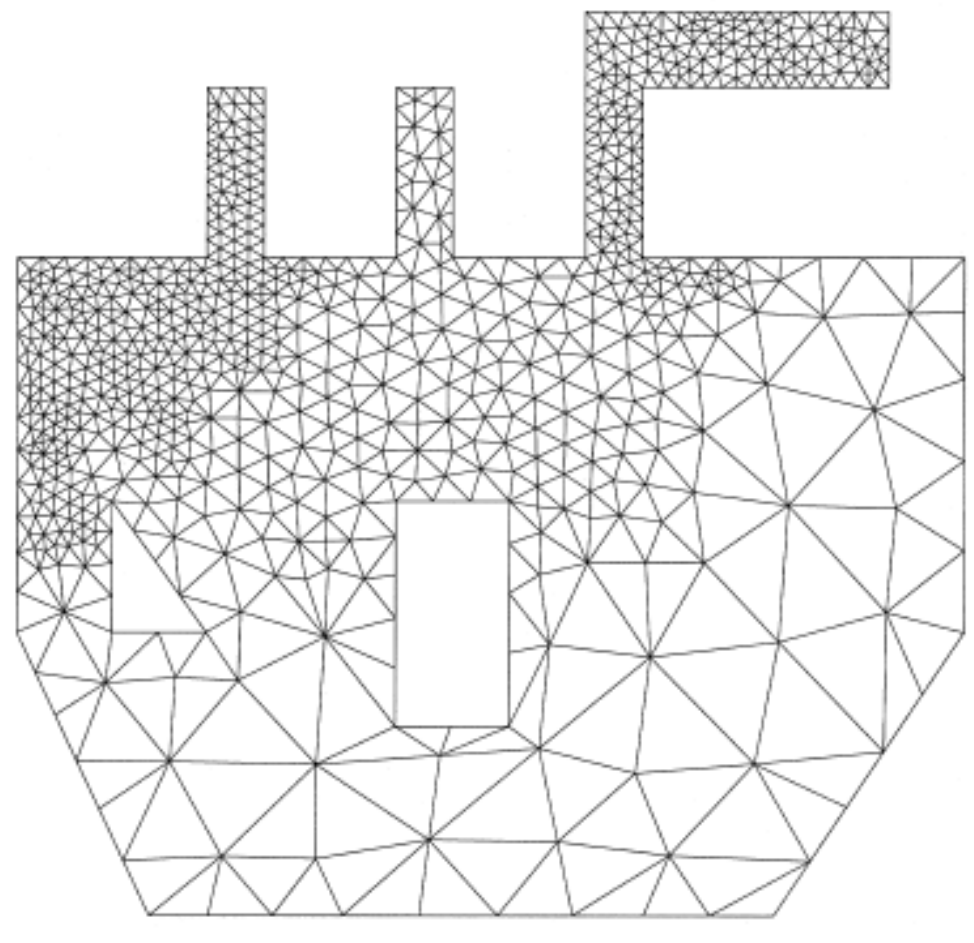

Figure 4. Mesh of domain in Figure 3

Figure 5 depicts the same mesh as Figure 4 after the mesh was passed through a “quadrilateralisation” subroutine. This subroutine computes the quadrilateral factors $\left\|\overrightarrow{\mathrm{f}}_{\mathrm{Q}}\right\|$ resulting from testing different combinations of triangles, and converts the latter to quadrilateral elements if a pre-specified quadrilateral factor limit is not exceeded. The time required for the latter process was 0.37 seconds, which is negligible in contrast to an overall time of 17.1 seconds for mesh generation. A maximum quadrilateral interior angle range of $90^{\circ} \pm 50^{\circ}$ was used to generate the mesh in Figure 5. As can be seen, the quality of a few of the quadrilaterals is poor, but this is due to the large maximum quadrilateral limit specified. 


\begin{tabular}{|c|c|c|}
\hline & $\begin{array}{c}\text { Triangular mesh } \\
\qquad\left(60^{\circ} \pm 30^{\circ}\right)\end{array}$ & $\begin{array}{l}\text { Hybrid mesh } \\
\left(90^{\circ} \pm 50^{\circ}\right)\end{array}$ \\
\hline$\left\|\overrightarrow{\mathrm{f}}_{\mathrm{T}}\right\|_{\min }$ (angle range) & $0.004\left(60^{\circ} \pm 0.1^{\circ}\right)$ & $0.004\left(60^{\circ} \pm 0.1^{\circ}\right)$ \\
\hline$\left\|\overrightarrow{\mathrm{f}}_{\mathrm{T}}\right\|_{\max }$ (angle range) & $1.013\left(60^{\circ} \pm 23.5^{\circ}\right)$ & $1.013\left(60^{\circ} \pm 33.5^{\circ}\right)$ \\
\hline$\left\|\vec{f}_{\mathrm{Q}}\right\|_{\min }$ (angle range) & & $1.523\left(90^{\circ} \pm 43.6^{\circ}\right)$ \\
\hline$\left\|\overrightarrow{\mathrm{f}}_{\mathrm{Q}}\right\|_{\max }$ (angle range) & & $1.620\left(90^{\circ} \pm 46.4^{\circ}\right)$ \\
\hline$\left\|\overrightarrow{\mathrm{f}}_{\mathrm{T}}\right\|_{a v}$ (angle range) & $0.376\left(60^{\circ} \pm 12.4^{\circ}\right)$ & $0.479\left(90^{\circ} \pm 15.8^{\circ}\right)$ \\
\hline$\left\|\overrightarrow{\mathrm{f}}_{\mathrm{Q}}\right\|_{a v}$ (angle range) & & $1.552\left(90^{\circ} \pm 44.4^{\circ}\right)$ \\
\hline Factor calculation time (s) & 0.21 & 0.37 \\
\hline Total CPU time (s) & 13.4 & 17.1 \\
\hline
\end{tabular}

Table 1. Quality factors and time taken for the domain in Figure 3

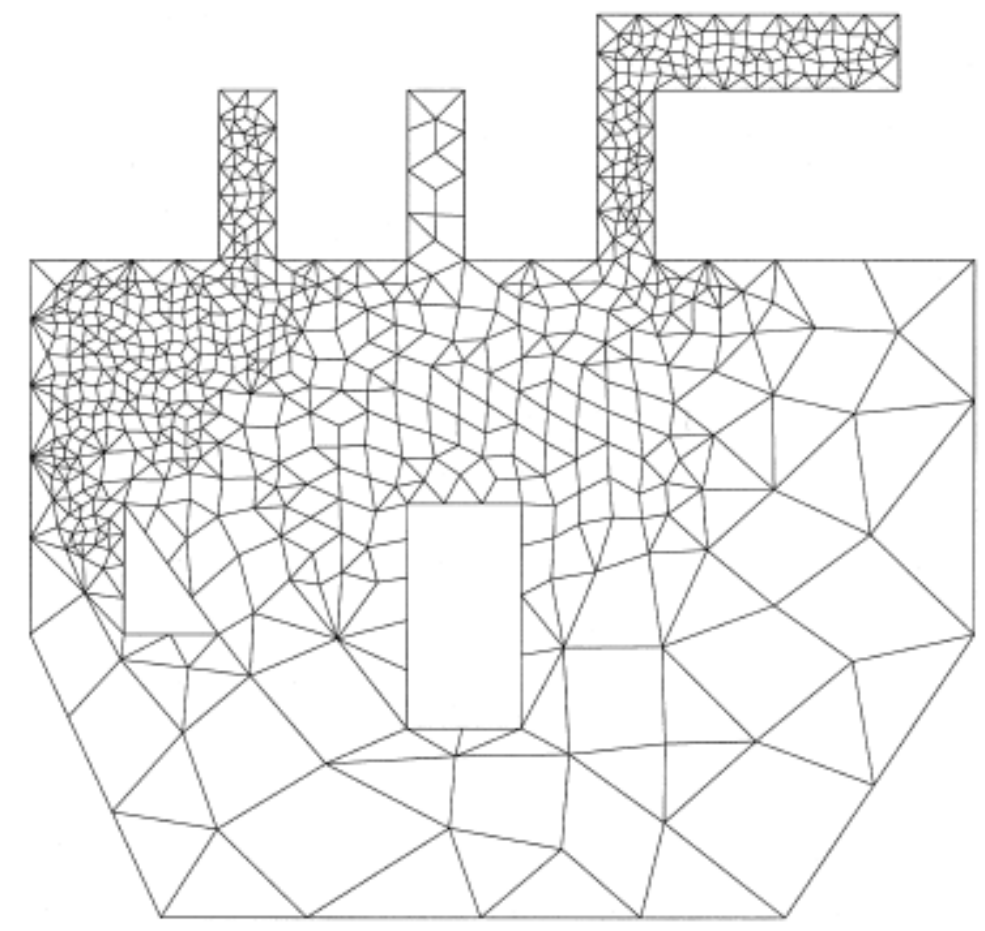

Figure 5. Hybrid mesh with maximum interior angles of $90^{\circ} \pm 50^{\circ}$

The domain shown in Figure 6(a) represents a typical practical problem with different materials and an opening such as an underground tunnel, and has been meshed into Figure 
6(b). Materials A and C have been meshed as mixed element materials, with material B having only triangles. The use of the new distortion factors to control the quality of generated elements has ensured the production of near-equilateral and quadrilateral elements.

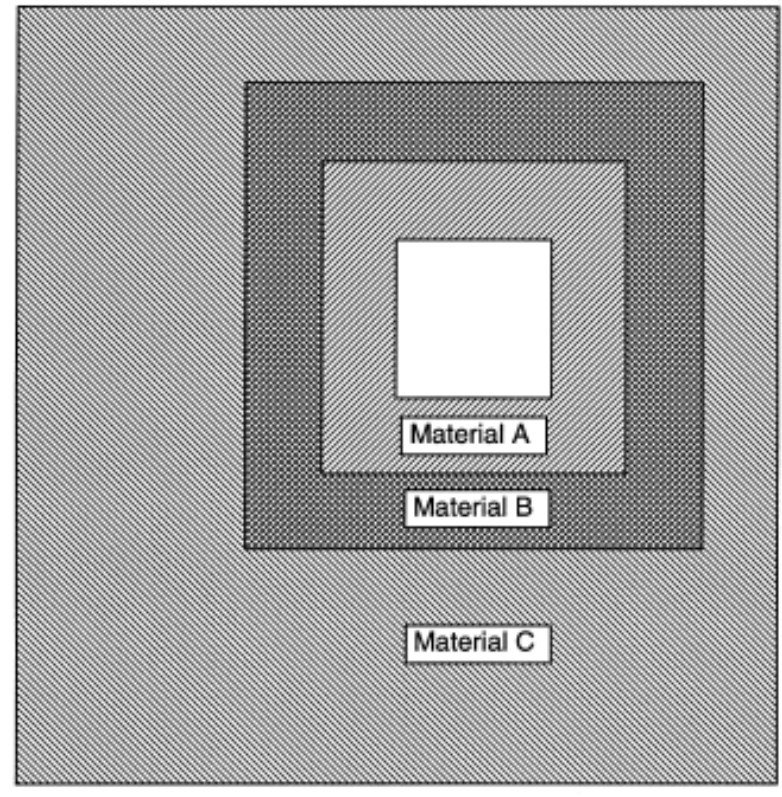

(a) Original domain

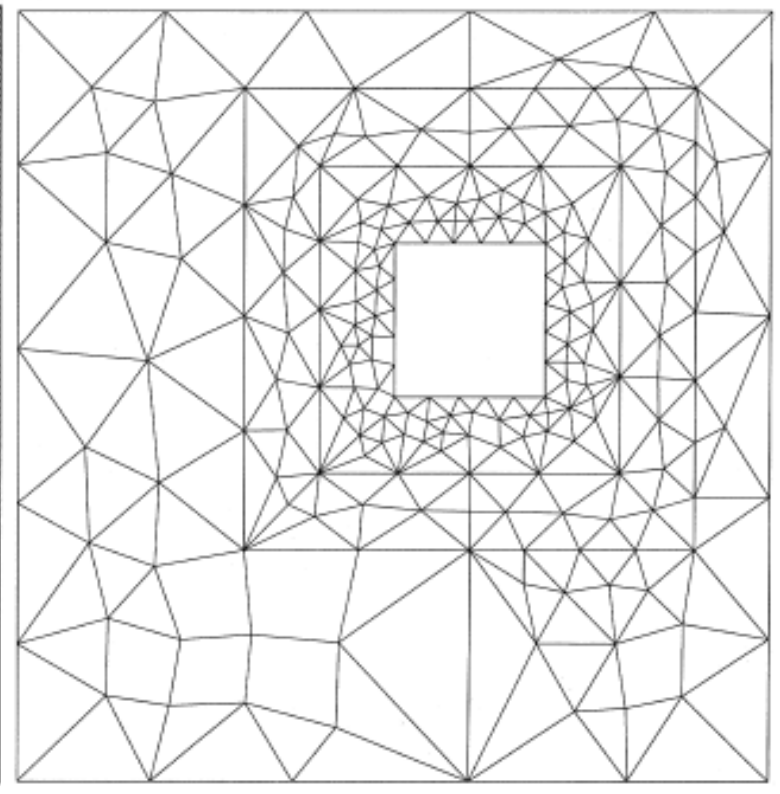

(b) Hybrid mesh

Figure 6. Opening in a multi-material domain

\section{CONCLUSIONS}

The concept of developing an element distortion factor based on interior angles only rather than lengths is a logical step for density-based mesh generators. The latter would involve mechanisms that ensure that the elements' aspect ratios are optimum, whereas angles have to be compared in some way. The new factors, $\left\|\overrightarrow{\mathrm{f}}_{\mathrm{T}}\right\|$ and $\left\|\overrightarrow{\mathrm{f}}_{\mathrm{Q}}\right\|$, serve that purpose by unifying three and four angles respectively into one parameter. These distortion factors can be easily extended to three-dimensional problems by computing the same factors using the interior angles for each plane. Four triangular factors would thus result for tetrahedral elements, while six quadrilateral factors for hexahedrals. In addition, advantage can be taken of neighbouring elements, which have the same distortion factor values across common faces, thus reducing the number of checks required, and in turn computation time. The time taken to 
check the quality of elements is very small compared to the mesh generation time itself, and time taken to run a 3D finite element analysis. The new distortion factor is thus a useful addition to the tools required to ensure the generation of good quality finite elements.

\section{REFERENCES}

[1] S. H. Lo, Generating quadrilateral elements on plane and over curved surfaces, Computers and Structures, 1989, 31, 421-426.

[2] S. H. Lo and C. K. Lee, On using meshes of mixed element types in adaptive finite element analysis, Finite Elements in Analysis and Design, 1992, 11, 307-336.

[3] J. Robinson, Some new distortion measures for quadrilaterals, Finite Elements in Analysis and Design, 1987, 3, 183-197.

[4] J. Robinson, Distortion measures for quadrilaterals with curved boundaries, Finite Elements in Analysis and Design, 1988, 4, 115-131.

[5] S. H. Lo, A new mesh generation scheme for arbitrary planar domains, International Journal for Numerical Methods in Engineering, 1985, 21, 1403-1426.

[6] T. S. Lau and S. H. Lo, Finite element mesh generation over analytical curved surfaces, Computers and Structures, 1996, 59, 301-309.

[7] J. Z. Zhu, O. C. Zienkiewicz, E. Hinton, and J. Wu, A new approach to the development of automatic quadrilateral mesh generation, International Journal for Numerical Methods in Engineering, 1991, 32, 849-866. 
[8] S. H. Lo, Automatic mesh generation and adaptation by using contours, International Journal for Numerical Methods in Engineering, 1991, 31, 689-707.

[9] R. V. Nambiar, R. S. Valera, K. L. Lawrence, R. B. Morgan, and D. Amil, An algorithm for adaptive refinement of triangular element meshes, International Journal for Numerical Methods in Engineering, 1993, 36, 499-509.

[10] S. H. Lo and C. K. Lee, Generation of gradation meshes by the background technique, Computers and Structures, 1994, 50, 21-32.

[11] D. S. Burnett, Finite element analysis - from concepts to applications, Addison-Wesley Publishing, Massachusetts (USA), 1988, Pages.

[12] J. E. Akin, Finite elements for analysis and design, Academic Press Ltd., London (UK), 1994, Pages. 